\title{
Research Paper: Assessment of Dynamic Stability During Walking in Below-knee Amputation
}

\author{
Keyvan Sharifmoradi $^{1}$, Mostafa Kamali $^{* *}$ Q, Mohammad Taghi Karimi² \\ 1. Department of Physical Education and Sport Sciences, Faculty of Humanities, University of Kashan, Kashan, Iran. \\ 2. Department of Orthotics and Prosthetics, School of Rehabilitation, Musculoskeletal Research Center, Isfahan University of Medical Sciences, Isfahan, Iran.
}

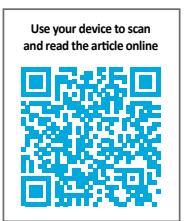

Crtation Sharifmoradi K, Kamali M, Taghi Karimi M. Assessment of Dynamic Stability During Walking in Below-knee Amputation. Physical Treatments. 2019; 9(2):77-84. http://dx.doi.org/10.32598/ptj.9.2.77

d tof" http://dx.doi.org/10.32598/ptj.9.2.77

(C) 08

Article info:

Received: 11 Oct 2018

Accepted: 27 Feb 2019

Available Online: 01 Apr 2019

Keywords:

Dynamic stability, Amputation, Margin of stability, Center of Mass

\section{A B S T R A C T}

Purpose: Dynamic stability reduces in patients with unilateral amputation; so, it is important to recognize changes in balance during walking. This study aimed to investigate the dynamic stability of unilateral below-knee amputation during walking.

Methods: The present study was a quasi-experimental study, in which 10 patients with knee amputation and 10 healthy young people participated. The variables of the present study included spatiotemporal variables, Center of Mass (COM) variables in anteroposterior, and mediolateral directions, as well as stability margin variables in anteroposterior and mediolateral directions. The COM and marginal stability variables were extracted through Visual 3D software. The data were analyzed by independent and dependent t-tests, using SPSS V. 22 at a significance level of 0.05 .

Results: The mean difference between COM and the COP in the amputation group in the frontal plane on both sound and prosthetic sides was significantly higher than those in the healthy group $(\mathrm{P}=0.01)$. The difference in COM and COP in the toe-off phase of the amputation group in the sagittal plane on the prosthetic side was significantly less than that in the healthy group $(\mathrm{P}<0.01)$.

Conclusion: The amputees suffer from poor dynamic stability during walking; so, they increase the positive margin of stability in the frontal plane and decrease the negative margin of stability in the sagittal plane. These patients decreased the excursion of COM in the sagittal and frontal plane, thereby using different gait patterns. However, the threats of falling in these patients are more than that of the healthy ones. Therefore, it is recommended to improve the dynamic stability of these patients in the frontal plane.

\section{* Corresponding Author:




\section{Highlights}

- The excursion of the center of mass in the sagittal plane was lower than that in the healthy group, but it was higher in a frontal plane compared to the healthy group.

- Below-knee amputation group walks with an increased margin of stability in the frontal plane.

- The decreased dynamic of stability can endanger the BKA group at the risk of falling.

\section{Plain Language Summary}

Dynamic stability changes during walking in the Below-knee Amputation (BKA) group compared to the healthy group and increase the risk of falling in these patients; so, we aimed to investigate the dynamic stability of this group during walking. Walking speed and stride length decreased in patients with BKA. To increase their stability during gait, these patients increase step width. Because of the defection of the ankle joint of the prosthetic leg, these patients cannot apply enough force in the propulsion phase of gait. So, the stride length of these patients also decreases. These changes during gait can decrease dynamic stability and endanger them at the risk of falling; thus, using appropriate prosthesis with active ankle and strengthening muscles can help these patients and prevent falling.

\section{Introduction}

ower limb amputation changes walking kinematic and kinetic variables and increases the risk of falling [1-4]. There is a lot of sensory and motor impairments in these patients while walking, which leads to an increased risk of falling [5]. Most of the falls occur during activities that involve the whole body, such as walking [6]. Restoring stability is very important during walking on a smooth or a rough surface, and up and down the steps. Therefore, examining walking and its affecting factors are important.

Humans are more unstable in the Mediolateral (ML) plane while walking, and more falls occur during walking, especially walking at unsustainable levels. Individuals with unilateral amputation appear to be more unstable than the healthy subjects, as they experience more falls compared to the healthy subjects [7]. Besides, patients with lower-limb amputations lose ankle function and active control over ankles joints [8].

By evaluating the dynamic stability of young people with transtibial amputation in an unstable environment, Beurskens et al. observed a greater instability in the prosthetic leg and showed that people with one-sided amputation had more dynamic instability than the healthy individuals, especially in the prosthetic leg [9]. Other studies also showed asymmetry in muscular force and joint contact force on a healthy and prosthetic side in these patients, which affected dynamic stability during walking. In ML plane, to increase stability, the Center of Mass (COM) must be placed within the margin of stability that leads to a positive stability margin $[1,3,10]$ While walking, people can adapt COM movements to control the margin of stability [11]. Recent investigations examined the movements of the COM while walking to assess the dynamic stability in different walking conditions, as well as the effect of side-to-side deviation on the walking of the healthy subjects [12]. These studies also reported that the mean stability margin of unstable walking in the healthy subjects and the stability margin variability significantly increased [13]. Thus, the stability of walking during unstable conditions could be examined by examining the margin of stability during walking.

People with upper limb amputations have shown more margin of stability in the prosthetic leg than the healthy one [14]. People with lower-limb amputations, like healthy people, increase their margin of stability against unstable conditions [15]. In the heel contact phase of walking, the difference between COM and the base of support was higher in people with unilateral amputation compared to the healthy people [15]. Although the relationship between the COM and the base of support has been studied, the relationship between the COM and the Center of Pressure (COP) in the lower limb amputations has been somehow overlooked.

It is important to know how these patients re-establish dynamic stability after transtibial amputation. This information helps us to improve their walking performance. Earlier studies investigated the degree of instability in unilateral amputations while walking on unstable surfaces and only examined the dynamic stability in the ML plane. Others cal- 
culated the margin of stability by marking the differences between COM and lateral malleus. However, in this study, the difference between COM and COP was considered as a margin of stability.

Little research assessed the dynamic stability in three planes; sagittal, frontal, and horizontal. A comprehensive examination of COM changes during walking will reveal more features of dynamic stability in unilateral Below-knee Amputation (BKA). Besides, research has been conducted to predict dynamic stability in young people, and research on older adults is limited. Thus, it is necessary to evaluate the dynamic stability of older individuals with BKA. In the present study, middle-aged people with BKA were studied. Therefore, this study aimed to examine the dynamic stability during walking in BKA.

\section{Materials and Methods}

Ten middle-aged people with BKA (with the Mean \pm SD height, weight, and age of $173.87 \pm 3.64 \mathrm{~cm}, 77.34 \pm 10.10$ $\mathrm{kg}$, and $48.0 \pm 2.98$ years, respectively) and 10 healthy young people (with the Mean \pm SD height, weight, and age of $168.6 \pm 27.17 \mathrm{~cm}, 78.83 \pm 26.93 \mathrm{~kg}$, and $25.28 \pm 2.33$ years, respectively) participated in this study. All subjects were selected and sampled after referral to the Rehabilitation Clinic of the Department of Orthotics and Prosthetics, Faculty of Rehabilitation, University of Isfahan.

The inclusion criteria were male gender, BKA, no history of major surgery or any disease affecting walking skills, healthy atrium, independent walking ability, the absence of any neurological or orthopedic disorder in a sound side, and the use of below-knee prosthetics for more than five years. The patients would be excluded from the study if there was an operation that disturbed the patients while standing and walking [2]. After explaining the goals and methods of the research for the subjects, they signed a consent form.

In record the data, first of all, the cameras and force plates were calibrated. Then, the subjects were asked to walk in the walking path. Barefoot walking was performed and repeated five times by each subject, and the average of three repetitions for each subject was calculated. A 30-second rest was considered between two repetitions for each subject.

The kinematic data were recorded by the Qualisys motion analysis system (manufactured by Qualisys; Switzerland) with a frequency of $100 \mathrm{~Hz} ; 20$ markers reflected infrared light with a diameter of $14 \mathrm{~mm}$ attached to the body subjects. Markers were attached to the anterior superior iliac spine, posterior superior iliac spine, medial and lateral epicondyles, heel, the first and fifth metatarsal head, and ac- romioclavicular joint on both right and left sides. Also, 16 cluster markers were attached to the legs and thighs of the left and right by the straps, as well. The placement of markers on the body was based on the protocol approved by the University of Strathclyde.

The kinetic data were also recorded by a Kistler force plate $(600 \times 500 \mathrm{~mm}$, model AA 9260 , manufactured by Kistler Co. Switzerland). Markers were reconstructed in Qualisys Track Manager Software and, then, transferred to Visual 3D (C-motion Inc.) software. The data collection frequency was $120 \mathrm{~Hz}$. The data were filtered by a low-pass filter with a frequency of $10 \mathrm{~Hz}$ [16]. A 9-segmental model was created to determine the movements of the COM and COP. The data of the center of foot pressure in the sagittal and frontal planes, as well as the data on the COM, were extracted from Visual 3D software.

The variables of the present research are spatiotemporal variables of walking (walking speed and stride length), the differences between COP and COM on the frontal plane, as well as in the sagittal plane, the excursion of COM in sagittal, frontal, and horizontal planes. The COM data were normalized to the length of the lower limb height in a vertical, step width in the frontal plane, and stride length in the sagittal plane. The difference between COM and COP on the sagittal and frontal planes was normalized to the height of the lower limb.

The sum of the differences between the point of the COM and the pressure on each leg divided by the number of points equaled the mean difference between the $\mathrm{COP}$ and the COM on the frontal plane. The margin of stability was defined as the difference between the center of the foot pressure and the COM in the ML and Anteroposterior (AP) plane. If the COM was placed inside the center of the foot pressure, the margin of stability would be positive, and if the COM were outside the center of the foot pressure, the margin of stability would be negative [10]. The data were analyzed by SPSS V. 22 (SPSS Inc., Chicago, IL), using the independent and dependent t-tests at a significant level of $<0.05$.

\section{Results}

Table 1 presents the demographic variables of the subjects. Table 2 presents the mean and standard deviation of spatiotemporal variables in both sound and prosthetic sides of patients with BKA.

As can be seen, the Mean \pm SD step width of the BKA group was $15.22 \pm 4.52 \mathrm{~cm}$, which was significantly higher than the healthy group $(\mathrm{P}=0.00)$. The walking speed 
Table 1. Demographic characteristics of the amputees

\begin{tabular}{|c|c|c|c|c|c|c|}
\hline Patient & Cause & Prosthetic & Prosthesis Side & Height, $\mathrm{cm}$ & Weight, kg & Age, y \\
\hline 1 & Burn & Padlini, with sachs button, foam & Left & 175 & 77.8 & 45 \\
\hline 2 & Trauma & Modular with a single access, bush joint, foam & Left & 174 & 79.8 & 49 \\
\hline 3 & Trauma & Single access, gel system & Right & 178 & 78.4 & 55 \\
\hline 4 & Trauma & One-piece autobook & Right & 170 & 60.2 & 48 \\
\hline 5 & Trauma & Single access & Left & 167 & 80.5 & 49 \\
\hline 6 & Trauma & Prosthetics with sach and cuff strap & Left & 170 & 65.0 & 47 \\
\hline 7 & Trauma & Single & Left & 173 & 89.4 & 54 \\
\hline 8 & Trauma & Modular sach & Left & 174 & 69.3 & 43 \\
\hline 9 & Trauma & Single access, autobook & Right & 176 & 86.3 & 51 \\
\hline 10 & Trauma & Prosthetics with Sachs and cuff strap & Right & 176 & 88.3 & 52 \\
\hline & & Mean $\pm S D$ & & $173.2 \pm 3.4$ & $77.5 \pm 9.9$ & $49.3 \pm 3.8$ \\
\hline
\end{tabular}

and step length in both sides of the sound and prosthesis leg was $0.31 \mathrm{~cm} / \mathrm{s}$ and $0.32 \mathrm{~cm} / \mathrm{s}$, respectively, that was significantly lower than that of the healthy group $(\mathrm{P}=0.001)$. The step length on sound and prosthetic legs were $10 \mathrm{~cm}$ and $14 \mathrm{~cm}$ lower than the healthy group $(\mathrm{P}=0.001)$.

Table 3 presents the mean and standard deviation of the difference between the Center of Gravity (COG) and the COP of both healthy and the amputation groups in sagittal, frontal, and horizontal planes. As can be seen, the mean difference between the COG and the COP in the BKA group in the frontal plane on both sound and prosthetic sides was significantly higher than that of the healthy group $(\mathrm{P}=0.01)$. Also, in the heel contact phase of gait, the mean difference in COG and COP of the BKA group in the sagittal plane in both sound and prosthetic sides was significantly lower than that of the healthy group ( $\mathrm{P}=0.01)$. In the toe-off phase, the difference in the COG and COP of the BKA group on the sagittal plane in both sound and prosthetic sides was significantly lower than that of the healthy group $(\mathrm{P}<0.01)$.

Table 2. The Mean \pm SD of spatial time variables

\begin{tabular}{|c|c|c|c|c|c|}
\hline \multirow{2}{*}{ Side } & \multirow{2}{*}{ Variables } & \multicolumn{2}{|c|}{ Mean $\pm S D$} & \multirow{2}{*}{$\mathbf{t}$} & \multirow{2}{*}{ Sig. } \\
\hline & & Healthy Group & BKA Group & & \\
\hline \multirow[t]{2}{*}{ Sound and prosthetic leg } & Step width (cm) & $6.44 \pm 2.62$ & $15.22 \pm 4.52$ & 5.04 & 0.00 \\
\hline & Foot length $(\mathrm{cm})$ & $98.89 \pm 3.02$ & $95.11 \pm 4.94$ & -1.96 & 0.07 \\
\hline \multirow[t]{3}{*}{ Healthy } & Speed $(\mathrm{cm} / \mathrm{s})$ & $1.22 \pm 0.08$ & $8.67 \pm 0.89$ & 25.08 & 0.00 \\
\hline & Step length $(\mathrm{cm})$ & $131.06 \pm 5.91$ & $117.51 \pm 11.61$ & -3.11 & 0.01 \\
\hline & Foot length $(\mathrm{cm})$ & $98.89 \pm 3.02$ & $94.77 \pm 4.54$ & -1.82 & 0.10 \\
\hline \multirow[t]{2}{*}{ Prosthesis } & Speed $(\mathrm{cm} / \mathrm{s})$ & $1.22 \pm 0.08$ & $8.34 \pm 1.03$ & 20.67 & 0.00 \\
\hline & Step length $(\mathrm{cm})$ & $131.06 \pm 5.96$ & $115.34 \pm 11.67$ & -3.60 & 0.000 \\
\hline
\end{tabular}


Table 3. The Mean \pm SD of the COG and COP differences between healthy and BKA groups (normalized to foot length)

\begin{tabular}{|c|c|c|c|c|c|}
\hline \multirow{2}{*}{ Side } & \multirow{2}{*}{ COG and COP Difference } & \multicolumn{2}{|c|}{ Mean $\pm S D$} & \multirow{2}{*}{$\mathbf{t}$} & \multirow{2}{*}{ Sig } \\
\hline & & Healthy Group & BKA Group & & \\
\hline \multirow{3}{*}{ Healthy } & Horizontal plan & $6.48 \pm 2.51$ & $8.79 \pm 1.89$ & 2.20 & 0.04 \\
\hline & Heel contact (Sagittal) & $29.56 \pm 5.19$ & $21.57 \pm 3.24$ & 3.91 & 0.00 \\
\hline & Toe-off (sagittal) & $32.35 \pm 4.32$ & $25.83 \pm 5.49$ & -3.91 & 0.01 \\
\hline \multirow{3}{*}{ Prosthesis } & Horizontal plan & $6.48 \pm 2.51$ & $9.32 \pm 1.12$ & 3.11 & 0.00 \\
\hline & Heel contact (Sagittal) & $29.56 \pm 5.19$ & $20.44 \pm 4.63$ & -3.93 & 0.00 \\
\hline & Toe-off (sagittal) & $32.35 \pm 4.32$ & $24.90 \pm 5.28$ & -3.28 & 0.00 \\
\hline
\end{tabular}

Table 4. The Mean $\pm \mathrm{SD}$ of the COM in both healthy and BKA groups

\begin{tabular}{|c|c|c|c|c|c|}
\hline \multirow{2}{*}{ Variable } & \multirow{2}{*}{ Page } & \multicolumn{2}{|c|}{ Mean $\pm S D$} & \multirow{2}{*}{$\mathbf{t}$} & \multirow{2}{*}{ Sig } \\
\hline & & Healthy Group & BKA Group & & \\
\hline \multirow{3}{*}{ Mass center $(\mathrm{cm})$} & Sagittal & $116.93 \pm 14.06$ & $146.21 \pm 14.71$ & 4.31 & 0.00 \\
\hline & Frontal & $4.79 \pm 1.75$ & $6.82 \pm 0.99$ & 3.03 & 0.01 \\
\hline & Horizontal & $3.38 \pm 0.61$ & $3.57 \pm 0.38$ & 0.77 & 0.45 \\
\hline \multirow{3}{*}{ COM (normalized) } & Sagittal (step length) & $111.77 \pm 12.71$ & $100.14 \pm 14.15$ & -1.84 & 0.09 \\
\hline & Frontal (step width) & $101.20 \pm 86.67$ & $48.84 \pm 13.38$ & -1.84 & 0.08 \\
\hline & Horizontal (foot length) & $3.42 \pm 0.64$ & $3.76 \pm 0.46$ & 1.28 & 0.22 \\
\hline
\end{tabular}

Table 4 presents the mean and standard deviation of the COM in both healthy and BKA groups with and without normalization. As can be seen, the displacement of the $\mathrm{COM}$ in a non-normalized state on the sagittal plane in the BKA group is $11.93 \mathrm{~cm}$, which is significantly lower than that of the healthy group $(\mathrm{P}<0.01)$. The displacement of the COM in the non-normalized state on the frontal plane was $6.82 \mathrm{~cm}$ in the BKA group, which was significantly higher than that in the healthy group $(\mathrm{P}=0.01)$. There was no significant difference between groups in the horizontal plane $(\mathrm{P}=0.45)$. The normalized displacements of the COM in the sagittal $(\mathrm{P}=0.09)$, frontal $(\mathrm{P}=0.08)$, and horizontal planes $(\mathrm{P}=0.22)$ were similar between both groups, and no significant differences were observed.

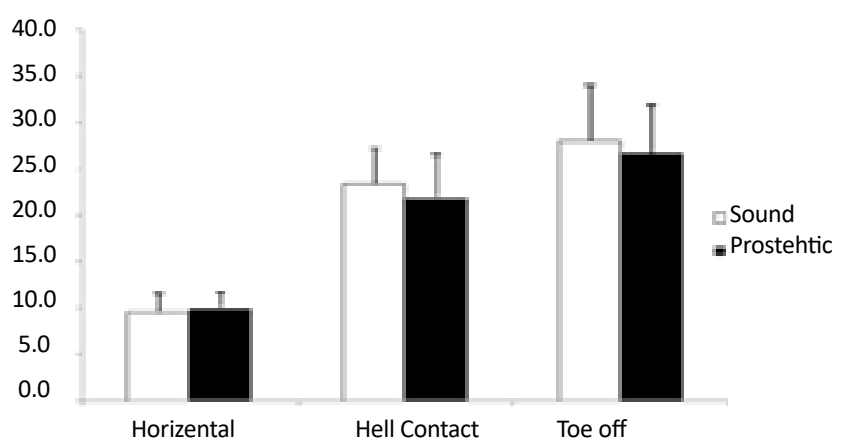

PHYSICAL TREA $\mid$ MENTS

Figure 1. The mean and standard deviation of COG difference and pressure center between the two sides of the BKA group 
Figure 1 shows the mean and standard deviation of the difference between the COG and the COP comparing the sound and prosthesis legs of the BKA group. As can be seen, in the heel contact phase, there was no significant difference between the COG and COP in the transverse plane. In the toe-off phase, there were no significant differences between the two legs in BKA group in the sagittal plane.

\section{Discussion}

Individuals with BKA have a lot of sensory and motor impairments during walking, which leads to an increased risk of falling for them so many experience falling during walking [4]. Falling is associated with the risk of fractures and imposing heavy hospital costs in these patients. It is important to identify the stability mechanism and the assessment of the weaknesses of the walking stability of patients with BKA. This knowledge will help the therapists to improve the dynamic stability of these patients. Therefore, this study aimed to investigate the dynamic stability of BKA in the three planes. The results of this study showed that the non-normalized lateral displacement of the COG and the lateral margin of stability on both legs in the BKA group was significantly higher than that of the healthy group. In both heel contact and toe-off phases of gait, the difference between the COM and the COP in the anterior plane of the BKA group was significantly lower than that in the healthy group.

The results of this study showed that the BKA group had lower walking speed, lower step length, and higher step width compared to the healthy group. These results are consistent with other researchers' findings, stating that lower limb amputations have lower speed, lower step lengths, and more steps width $[15,17]$. When people with amputations try to walk with prostheses, they feel unstable; so, they choose walking with a shorter step length and a higher step width. Given that the healthy group was younger than the patient group, can we infer that age affects the outcome? It is worth mentioning that the difference in age between patients and young people does not cause a significant difference in their walking. Also, because of the normalization of the step length to the length of the lower limb and the step width to the width of the pelvis, the age difference becomes largely ineffective.

The results also showed that the difference between the $\mathrm{COM}$ and the center of foot pressure is similar between sound and prosthetic legs in the BKA group, which is consistent with the studies by Gate et al. and Beltran et al. $[15,18]$. Although there was no significant difference between the sound and prosthetic legs, the difference in $\mathrm{COM}$ and the COP on the prosthetic leg was less in the sound leg. This difference may be a result of the strategy of gait in these patients. Because of the lack of ankle strategy, these patients have to produce more force by the sound leg in the stance phase to put the prosthesis leg in the appropriate place; so, they put more pressure on the sound leg [19].

The results showed that the BKA group had a higher lateral margin of stability than the healthy group, while the mean lateral margin of stability in the BKA group was significantly higher than that of the healthy group. The results of this study were consistent with the results of the research by Beltran et al. [18]. They showed that subjects with unilateral amputations had a greater lateral margin of stability than the healthy ones. It is also consistent with the results of Hak et al., Young and Dingwell, and McAndrew et al. [20-22]. They also showed that the step width of patients with BKA increased, leading to an increase in the margin of stability in these patients.

This finding indicates that people with unilateral amputation of the lower limb are unstable in the frontal plane compared to the healthy group. The ankle is a complex joint and does various movements including abduction, supination, and pronation [23]. Perhaps, patients with BKA showed greater instability in the frontal plane in the prosthetic side because of the inability to perform various ankle movements, weakness in the control of ankle, sensory impairment in the limbs, and the loss of sensory information required for stability during walking [24]. The BKA group moves COM in a smaller range, but walks with the wider step width and more margin of stability in the frontal plane, thereby trying to increase their dynamic stability while walking. Hak et al. showed that an increase in step width would increase the margin of stability [25]. Young et al. also showed that even when young healthy people are faced with the challenge of instability during walking, they try to increase their margin of stability so that the dynamic stability of walking increases [21].

The differences between the COM and COP in heel contact and toe-off phases on both sound and prosthetic legs of the amputation group were significantly lower than those of the healthy group. In the sense that the COM in the healthy group was significantly closer to the COP in both heel contact and toe-off phases, putting the COM away from the COP in the toe-off phase requires a strong "push-off'. A high percentage of push-off power is because of the function of the ankle plantar-flexors muscles in the stance phase, while the amputation group is unable 
to produce sufficient force the same as the healthy group because of the lack of ankle function; thus, they produce lower power at the push-off phase [26]. Therefore, it is impossible to move COM at a further distance from the COP, which reduces the amount of anterior progression in the BKA group; the results of this study also showed that the step length of the BKA group was less than the healthy group.

These results indicate that patients with BKA use a different strategy during gait, which controls the placement of the foot on the ground. They also control the movements of the COM differently from the healthy people. These changes in gait result from limb deficiency, the lack of ankle performance, and deficiency of afferent sensory information, which is needed to controlling dynamic stability during gait.

The amputees suffer from poor dynamic stability during walking; so, they increase the positive margin of stability in the frontal plane and decrease the negative margin of stability in the sagittal plane. These patients decreased the excursion of COM in the sagittal and frontal planes, thereby using different gait patterns. However, the threats of falling for these patients are more than that of the healthy ones. Therefore, it is recommended to improve the dynamic stability of these patients in the frontal plane.

\section{Ethical Considerations}

\section{Compliance with ethical guidelines}

All subjects read and signed consent form before participated in study. They were allowed to discontinue the study whenever they wished, and if desired, the results of the research would be available to them.

\section{Funding}

Research Deputy of Isfahan University of Medical Sciences has paid all the costs of the research.

\section{Authors' contributions}

Conceptualization, methodology, resources: All authors; Investigation, Funding Acquisition: Mostafa Kamali, Mohammad Taghi Karimi; Writing-original draft, Writing-review \& editing: Keyvan Sharifmoradi, Mohammad Taghi Karimi; Supervision: Mohammad Taghi Karimi.

\section{Conflict of interest}

The authors declared no conflict of interest.

\section{Acknowledgments}

The authors would like to gratitude the Musculoskeletal Research Center and Research Deputy of Isfahan University of Medical Sciences.

\section{References}

[1] Sharifmoradi K, Kamali M, Karimi MT. [Effect of hemipelvectomy amputation on kinematics and muscle force generation of lower limb while walking (Persian)]. Journal of Rehabilitation (USWR). 2017; 18(2):122-31. [DOI:10.21859/ jrehab-1802120]

[2] Sharifmoradi K, kamali M, Karimi M. [Comparison of ground reaction forces components on sound and prosthetic legs in trands-tibial amputated individuals (Persian)] Iranian Journal of War and Public Health (IJWPH). 2016; 8(2):75-82.

[3] Sharifmoradi K, Kamali Ardekani M, Karimi MT. The interaction of knee, hip and 15-s1 joint contact forces and spatiotemporal variables between sound and prosthetic leg in patients with unilateral below-knee amputation during walking. Journal of Rehabilitation Sciences and Research. 2018; 4(2):53-9. [DOI:10.30476/JRSR.2017.41119]

[4] Grumillier C, Martinet N, Paysant J, André JM, Beyaert C. Compensatory mechanism involving the hip joint of the intact limb during gait in unilateral trans-tibial amputees. Journal of Biomechanics. 2008; 41(14):2926-31. [DOI:10.1016/j. jbiomech.2008.07.018] [PMID]

[5] Miller WC, Speechley M, Deathe B. The prevalence and risk factors of falling and fear of falling among lower extremity amputees. Archives of Physical Medicineand Rehabilitation. 2001; 82(8):1031-7. [DOI:10.1053/apmr.2001.24295] [PMID]

[6] Tinetti ME, Doucette J, Claus E, Marottoli R. Risk factors for serious injury during falls by older persons in the community. Journal of the American Geriatrics Society. 1995; 43(11):1214-21. [DOI:10.1111/j.1532-5415.1995.tb07396.x] [PMID]

[7] Sinitksi EH, TerryK, Wilken JM, Dingwell JB. Effects of perturbation magnitude on dynamic stability when walking in destabilizing environments. Journal of Biomechanics. 2012; 45(12):2084-91. [DOI:10.1016/j.jbiomech.2012.05.039] [PMID]

[8] Viton JM, Mouchnino L, Mille M, Cincera M, Delarque A, Pedotti A, etal. Equilibrium and movement control strategies in trans-tibial amputees. Prosthetics and Orthotics International. 2000; 24(2):108-16. [DOI:10.1080/03093640008726533] [PMID]

[9] Beurskens R, Wilken JM, Dingwell JB. Dynamic stability of superior vs. inferior body segments in individuals with transtibial amputation walking in destabilizing environments. Journal of Biomechanics. 2014; 47(12):3072-9. [DOI:10.1016/j. jbiomech.2014.06.041] [PMID] [PMCID] 
[10] Hof AL, Gazendam MG, Sinke WE. The condition for dynamic stability. Journal of Biomechanics. 2005; 38(1):1-8. [DOI:10.1016/j.jbiomech.2004.03.025] [PMID]

[11] Hof AL. The 'extrapolated center of mass' conceptsuggests a simple control of balance in walking. Human Movement Science. 2008; 27(1):112-25. [DOI:10.1016/j.humov.2007.08.003] [PMID]

[12] Sharifmoradi K, Farahpour N, Karimi MT, Bahram A. Analysis of dynamic balance during walking in patients with Parkinson's disease \& able-bodied elderly people. Physical Treatments Journal Journal (PTJ). 2015; 4(4):191-8.

[13] McAndrew PM, Wilken JM, Dingwell JB. Dynamic stability of human walking in visually and mechanically destabilizing environments. Journal of Biomechanics. 2011; 44(4):644-9. [DOI:10.1016/j.jbiomech.2010.11.007] [PMID] [PMCID]

[14] Hof AL, van Bockel RM, Schoppen T, Postema K. Control of lateral balance in walking: Experimental findings in normal subjects and above-knee amputees. Gait \& posture. 2007; 25(2):250-8. [DOI:10.1016/j.gaitpost.2006.04.013] [PMID]

[15] Gates DH, Scott SJ, Wilken JM, Dingwell JB. Frontal plane dynamic margins of stability in individuals with and without transtibial amputation walking on a loose rock surface. Gait \& Posture. 2013; 38(4):570-5. [DOI:10.1016/j.gaitpost.2013.01.024] [PMID] [PMCID]

[16] Kadaba M, Ramakrishnan H, Wootten M, Gainey J, Gorton G, Cochran G. Repeatability of kinematic, kinetic, and electromyographic data in normal adult gait. Journal of Orthopaedic Research. 1989; 7(6):849-60. [DOI:10.1002/ jor.1100070611] [PMID]

[17] Lamoth CJ, Ainsworth E, Polomski W, Houdijk H. Variability and stability analysis of walking of transfemoral amputees. Medical Engineering \& Physics. 2010; 32(9):1009-14 [DOI:10.1016/j.medengphy.2010.07.001] [PMID]

[18] Beltran EJ, Dingwell JB, Wilken JM. Margins of stability in young adults with traumatic transtibial amputation walking in destabilizing environments. Journal of Biomechanics. 2014; 47(5):1138-43. [DOI:10.1016/j.jbiomech.2013.12.011] [PMID] [PMCID]

[19] Kamali M, Sharif-Moradi K, Tahmasebi A, Jabal-Ameli K. Effect of below knee prosthesis on muscle force production and joint contact forces of knee and hip joints during walking in amputees. Iranian Journal of War and Public Health. 2017; 9(2):79-84. [DOI:10.18869/acadpub.ijwph.9.2.79]

[20] Hak L, Houdijk H, Steenbrink F, Mert A, van der Wurff $\mathrm{P}$, Beek PJ, et al. Stepping strategies for regulating gait adaptability and stability. Journal of Biomechanics. 2013; 46(5):905-11. [DOI:10.1016/j.jbiomech.2012.12.017] [PMID]

[21] Young PMM, Wilken JM, Dingwell JB. Dynamic margins of stability during human walking in destabilizing environments. Journal of Biomechanics. 2012; 45(6):1053-9. [DOI:10.1016/j.jbiomech.2011.12.027] [PMID] [PMCID]

[22] Young PMM, Dingwell JB. Voluntary changes in step width and step length during human walking affect dynamic margins of stability. Gait \& Posture. 2012; 36(2):219-24. [DOI:10.1016/j.gaitpost.2012.02.020] [PMID] [PMCID]

[23] Levangie PK, Norkin CC. Joint structure and function: A comprehensive analysis, $5^{\text {th }}$ edition. Philadelphia: F.A. Davis Company; 2011.
[24] Powers CM, Torburn L, Perry J, Ayyappa E. Influence of prosthetic foot design on sound limb loading in adults with unilateral below-knee amputations. Archives of Physical Medicine and Rehabilitation. 1994; 75(7):825-9. [PMID]

[25] Hak L, Houdijk H, Steenbrink F, Mert A, van der Wurff P, Beek PJ, et al. Speeding up or slowing down? gait adaptations to preserve gait stability in response to balance perturbations. Gait \& Posture. 2012; 36(2):260-4. [DOI:10.1016/j. gaitpost.2012.03.005] [PMID]

[26] Seroussi RE, Gitter A, Czerniecki JM, Weaver K. Mechanical work adaptations of above-knee amputee ambulation. Archives of Physical Medicine and Rehabilitation. 1996; 77(11):1209-14. [DOI:10.1016/S0003-9993(96)90151-3] 